\title{
HANDS-ON EDUCATION WiTHOUT THE HANDS-ON? AN APPROACH TO ONLINE DELIVERY OF A SENIOR LAB COURSE IN Chemical EngineERING WhILE MAINTAINING KeY LEARNING OUTCOMES
}

\author{
Roza Vaez Ghaemi ${ }^{1,2}$ and Gabriel Potvin ${ }^{l}$ \\ ${ }^{1}$ Department of Chemical and Biological Engineering, University of British Columbia \\ ${ }^{2}$ School of Biomedical Engineering, University of British Columbia \\ ghaemi@chbe.ubc.ca, gabriel.potvin@ubc.ca
}

\begin{abstract}
The senior chemical engineering lab course at $U B C$ follows an open-ended problem-based lab (PBL) framework, which has been shown in previous work to improve self-reported learning outcomes in students. Given the hands-on experiential components of lab-based courses in general, their shift to remote delivery, such as the one required by the COVID-19 pandemic, is challenging. Shifting a PBL course to a virtual setting while maintaining its more open-ended learning outcomes is likely even more difficult. This paper outlines the design of an online senior PBL course in chemical and biological engineering, in which TAs, present in the lab, act as Operators and implement student-designed experimental plans over several weeks, while maintaining active virtual contact with their teams. Feedback from students on this experience, the particulars of the course design, and the perceived value of this approach, based on data collected from survey responses, is presented and compared to similar data collected for the latest in-person offering of the same course.
\end{abstract}

Keywords: Laboratory Education, Online Education, Problem-Based Learning

\section{INTRODUCTION}

Given the hands-on experiential components of labs, online learning has traditionally been limited to theoretical, lecture-based courses [1,2]. Lab courses, however, play a crucial role in engineering curricula as some of the key opportunities to facilitate the development of analytical skills and higher-order transferrable skills including critical-thinking, problem-solving and troubleshooting, teamwork, and technical communication $[3,4]$.

With the advent of the COVID-19 pandemic and the consequent move to online learning, laboratory courses faced the daunting challenge of adapting and re-imagining learning outcomes normally associated with hands-on work. Replicating core components of the lab experience, namely the hands-on manipulation, control and observation of phenomena and processes, in a non-trivial manner is difficult to do in an online setting [5,6], let alone as part of active collaborative teams like those commonly associated with lab courses. This is particularly difficult in disciplines like chemical and biological engineering in which labs typically involve hazardous chemicals and large stationary unit operations that are difficult to scale or replicate in non-specialized facilities.

Solutions do exist, of course, in the form of custom simulations or recorded demonstrations, but these can be costly, remove most of the true experimentation component of these courses, and usually remove the need for troubleshooting or working with real (i.e. messy) data, which may eliminate valuable learning opportunities for engineering students. Some reports of approaches include, for example, a guided-inquiry activity used to engage students in an organic chemistry lab; edited videos of the experimental procedures in a analytical chemistry lab course [3], experiments performed at home using provided kits, virtual experiments using commercial software, or synchronous live lab demonstrations or video tutorials [7]. Although these approaches are successful in delivering content and providing opportunities to develop analytical skills, the extent to which students can actively engage with the material, and do so in a realistic way, is unclear.

In the Department of Chemical and Biological Engineering (CHBE) at the University of British Columbia (UBC), the $4^{\text {th }}$-year laboratory course (CHBE 464), under normal (in-person) circumstances, requires teams of students to perform multi-week open-ended, problembased laboratories (PBL). Open-ended industriallyrelevant problem statements consisting of design, optimization or investigative objectives are provided to student teams. In each of the course's two terms, teams design and execute sophisticated experimental plans themselves with very limited direction or instructor or TA 
involvement, and have opportunities to present, defend and revise their work at specific intervals. The hands-on experience combined with the independence provided and accountability for choices helps develop higher order troubleshooting, data analysis, and critical thinking skills. In previous work, the structure of this course was described in detail, and students reported this approach helped them not only develop their critical thinking and problemsolving skills, but also increase their confidence in their engineering abilities and feeling of agency and engagement in their education $[9,10]$.

In response to the pandemic and the resulting shift to online teaching, this lab course was redesigned for the 2020/2021 academic year to allow for its remote delivery while maintaining key learning outcomes associated with ownership and control of the work, open-ended problemsolving, troubleshooting, and the drafting of technical recommendations based on critical analyses of real data. Transferrable skills related to teamwork and technical communication were also emphasized compared to the regular course offering.

This paper outlines the specifics of this online course design, as well as feedback from students on the experience and their perceived value of this approach, based on data collected from survey responses and instructor observations. Suggestions for the implementation of this model in other contexts and for its improvement are also provided.

\section{PEDAGOGICAL APPROACH}

The PBL approach mimics the structure of real engineering projects, including preliminary project planning and consultation phases, risk assessment, resource allocation, experimental data collection, and the reporting and presentation of final results, recommendations, and conclusions. Low-stakes periodic review of work with feedback allows students to revise their work plans, immediately correct misconceptions and errors in judgement, and promotes creativity by removing penalties for those errors [8].

The pedagogical design of the problem-based laboratory is based on a socio-constructivist framework leveraging team-based experiential learning concepts. The objectives of the course are centered around "investigation", "design", "lifelong learning" and higherorder skills such as critical thinking and problem-solving [9]. In contrast with traditional labs where specific instructions or protocols are provided to students, who implement these instructions and are assessed in part on whether they obtained the expected results, in PBL approaches, students are asked to solve real, open-ended engineering problems and are assessed based on their thought processes, creative problem-solving, experimental design, and knowledge integration as applied to these practical scenarios.

In the online version, the open-ended PBL format puts added emphasis on communication, project management, and leadership. Specifically, the need to communicate with the process Operators (TAs performing the experiments) and provide them with justified technical instructions helps students to integrate theories and actively evaluate and refine their often initially flawed or incomplete experimental designs.

\section{COURSE DESIGN}

CHBE 464 is often described by students as the "lab Capstone". It is a stand-alone core course composed of wet and dry lab sessions focusing on experimental design, data analysis, and engineering decision-making. In previous work, the regular, in-person PBL lab course design was described in detail [10]; and was shown to develop student confidence in their engineer skills and sense of agency in their education [11].

In this revised course design developed for online delivery, each term teams of 4-5 students are assigned, based on expressed interest, to one of the eleven available experimental setups listed in Table 1, and provided with an investigative or optimization problem statement to frame the work to be performed. Each team is also assigned a Process Operator, a TA assigned to their experimental apparatus who performs the work requested by the team, according to provided instructions. The small number of Operators necessary to run this course (11 TAs divided over three large lab spaces) along with stringent safety protocols allowed for the following of established UBC and CHBE regulations for the safe in-person performance of the experiments.

Like before, student teams are responsible for devising and defending experimental plans spanning 10 weeks, complete with protocols and detailed instructions, to address their problem statement. Teams give a low-stakes (i.e. low grade value) virtual oral presentation to present their project proposals, which include detailed experimental designs, safety and environmental assessments, quality assurance plans, and a management

Table 1: Available experimental setups

\begin{tabular}{|l|}
\hline a. Biodiesel production from UBC waste cooking oil \\
\hline b. $\mathrm{CO}_{2}$ absorption in packed towers \\
\hline c. $\mathrm{CO}_{2}$ capture through gas hydrate formation \\
\hline d. Hydrocyclone separations of starch solutions \\
\hline e. Electrosynthesis of hydrogen peroxide \\
\hline f. CSTR/PFR reactor design \\
\hline g. Plate Distillation of industrial solvents \\
\hline h. Yeast fermentation for industrially-relevant products \\
\hline i. Three-phase catalytic reactor operation \\
\hline j. Microalgae culture for oil production \\
\hline k. Genetic engineering and culture of bacteria \\
\hline
\end{tabular}


plan for the term, and receive detailed feedback from the instructor that they incorporate in a more heavily weighted written proposal. The Operators then perform the work, exactly as requested, returning the generated data to students at specified intervals. This approach incidentally led to amusing scenarios in which Operators, all experienced researchers, had to follow experimental protocols that they knew were incorrect and would return flawed results to students (who then had to analyze these results and revise their protocols as part of the learning process). It should be noted that although TAs are instructed to follow students' instructions accurately and allow them to make mistakes, they also serve as coaches and help orient and guide student teams through the process and provide technical or operational details as needed to compensate for the lack of possible hands-on experimentation or troubleshooting. At the end of the experimental sessions, teams once again give a low-stakes virtual oral presentation before submitting a final report incorporating any feedback received.

The implementation of the experimental plan by the Operator alternates between a 'wet lab' one week, during which the experiments are performed according to student's work plans, and a 'dry lab' the following week during which teams review the data generated and revise the following week's work plan as necessary. During 'wet labs' students are in synchronous contact with the Operators, who have been instructed to defer to students for all key process decisions. At the end of the term, teams present their final results and a plan for the implementation of their proposed solution. Although the hands-on components inherent to lab courses are not (and cannot be) truly replaced in this online setting, the experience maintains the experimental design, critical analysis, and practical engineering judgement components of the original course, and allow students to maintain ownership and agency in the process, while adding valuable technical communication experience through the need to work closely with the Operator.

The periodic low-stakes presentations to inform the revision of work before higher-stakes submissions, periodic check-ins with the instructor (short 15 minute virtual meetings between the instructor and each team), and TA training outlining guidelines to grant students as much autonomy as possible and follow their directives, allow students to actively engage with the course material despite not physically being in the lab, while being supported in their investigations and learning process.

\section{METHODOLOGY}

Midway through the second term of this PBL lab course (at which point they had completed their first lab, and had completed the proposal stage of their second one) students were invited to complete an anonymous survey, on a purely voluntary basis, asking about their overall experience with both the PBL approach to lab education in general, and on this online lab course experience specifically. The data collected was analyzed, and the results were used to evaluate students' perception of the value of this experience, and also compared to results of a similar survey completed by the previous cohort of students that participated in the in-person delivery of this PBL course in the 2019/2020 academic year.

The survey was composed of two sections. The first section consisted of nine 5-point Likert-scale questions (1 = strongly disagree; 2 = disagree; $3=$ neither agree nor disagree; $4=$ agree $; 5=$ strongly agree) asking participants to evaluate their experience with the online version of the course, the effectiveness of the course as part of their training (their perception of knowledge and skill development), as well as their experience remotely working with their teammates and Operators. The second section of the survey was composed of seven optional open-ended text questions asking students to elaborate on some of their answers from the first section as well as comment on their perception of the PBL framework as an approach to lab education. The full list of questions is shown in Table 2. Responses to these open-ended questions were thematically analyzed.

The survey included specific statements or questions that were also included in a similar survey administered to the students that completed the in-person offering of the course during the previous year. These questions are indicated with an asterisk in Table 2. This allows some measure of comparison of student perceptions of the course based on the mode of delivery. Approximately $40 \%$ of the cohorts responded to the survey in both the 2019/2020 (inperson, $n=45 / 122$ ) and 2020/2021 (online, $n=42 / 101$ ) academic years. Statistical analysis and two-sided t-test of the numeric values were performed to estimate the significance of each year's results in terms of mean score and p-value.

For the in-person cohort (in 2019/2020), students were also encouraged to write an end-of-course reflection on their personal experience, so that more individual and qualitative feedback into any aspect of the course could be collected and evaluated to inform further course improvements. Responses to these open-ended questions were also thematically analyzed. Despite the wide variety of projects, the identified positive aspects of the course were very similar, focusing primarily on autonomy (words like freedom, choice, responsibility, independence), and opportunities for self-improvement (comments with phrases like valuable feedback, low risk grading, and opportunities for discussions). The very few negative comments were mostly related to time constraints and equipment restrictions, all of which are related to logistics of course delivery. Full results and their analysis of the 2019/2020 survey were presented in previous work [11]. 
Table 2: List of questions administered to students taking the online offering of the PBL lab course

\section{Section 1 - Likert-Scale Questions}

Please indicate your level of agreement with the following statements:

1. I thought this course (in the online format used this year) was worth taking.

2. I have learned a lot in this course."

3. I have developed useful skills in this course.*

4. I was interested in the subject matter of my assigned labs.*

5. I am more confident in my engineering skills after taking this course.*

6. Collaborating with, and running experiments through, the TAs was a positive experience.

7. I perceive online learning as a positive experience in general.

8. I found it easy to collaborate with my peers for this lab.*

9. If I could choose, I would do these labs in person instead of online.

\section{$\underline{\text { Section } 2 \text { - Open Text Questions }}$}

10. How was your experience in this ONLINE format of lab course (i.e. having TAs do the experiments)?

11. If given the choice would you prefer to do these labs in person? Why? If you prefer online labs, why? Be specific.

12. How was communication and workload managed/distributed among the team members? Is this different or the same as your previous in-person group work experiences?

13. What are your thoughts on this particular approach to lab instruction (problem-based, design your own experiments, proposal/final reports, oral presentations, etc)? Consider this irrespective of whether it is offered online or in-person.

14. Provide any other comment you wish to make about this course. Keep in mind you will also have the opportunity to officially evaluate the course and its instructor in UBC course evaluations in a few weeks.

* The question was also included in a previous survey administered to students that took the in-person format of PBL during the 2019/2020 academic year

\section{RESULTS AND DISCUSSION}

42 students responded to the survey, out of a class of 102. Only a subset of the data collected is presented in this paper, but the authors are happy discuss further or to share the full data set with the interested reader.

\subsection{Class grade distribution}

Student performance in the current online format was compared with that of the last in-person offering of the course. Figure 1 compares the final Term 1 marks of the student teams for 2019 (in-person format with 123 students enrolled) with those of the 2020 cohort (online format with 102 students enrolled). The letters refer to the lab subject matter listed in Table 1. For each lab there are 2 or 3 student teams working separately on the problem, with the exception of the yeast fermentation project $(h)$ which was performed by only one student team in 2019 and was not performed in 2020 due to logistical constraints. Although it is difficult to directly compare different cohorts of students in terms of performance, both instances of the course were overseen by the same instructor, who systematically reviewed each deliverable following the same rubrics and standards. Although additional data would be necessary to draw any firm conclusions about learning outcomes in one format or another, it should be noted that overall performance in the course remains high (average of 87\%) for both online and in-person formats. The unusually high average of the class is due to the aforementioned low-stakes opportunities for students to present their work and receive feedback before submitting the more important deliverables, allowing for revisions, sometimes extensive, of their drafts and consequent improvement of marks. It is interesting to note that the proportion of students in the upper grade tiers $(85 \%+)$ remains approximately the same for both versions of the course, but that a greater proportion of students fall in the lower grade tiers $(70-80 \%)$ for the online setting. It is difficult to draw firm conclusions about these observations, which could be the result of a slew of factors, that may or may not be attributable to the format (online or in-person) of the course. It seems, however, that students are consistently delivering on the learning objectives designed for in these courses.

\subsection{Feedback from the students}

A summary of the responses to the survey on the online delivery of the course is shown in Figure 2, with points of interest described here. 35 out of 42 survey respondents found the PBL lab in this online format was worth taking (Q1) and indicated positive experiences collaborating and running the experiments through the TAs (Q6). In Q11, responses suggest that in addition to the usual benefits associated with labs, students appreciated the lower time 
commitment (compared to in-person labs), the flexibility of the schedule, and the focus on the communication and analysis of their own unique data. Some students reported finding aspects of the course challenging, noting difficulties devising workplans for the Operator due to a lack of previous experience with the specific experimental setups (a challenge also reported for in-person offerings),
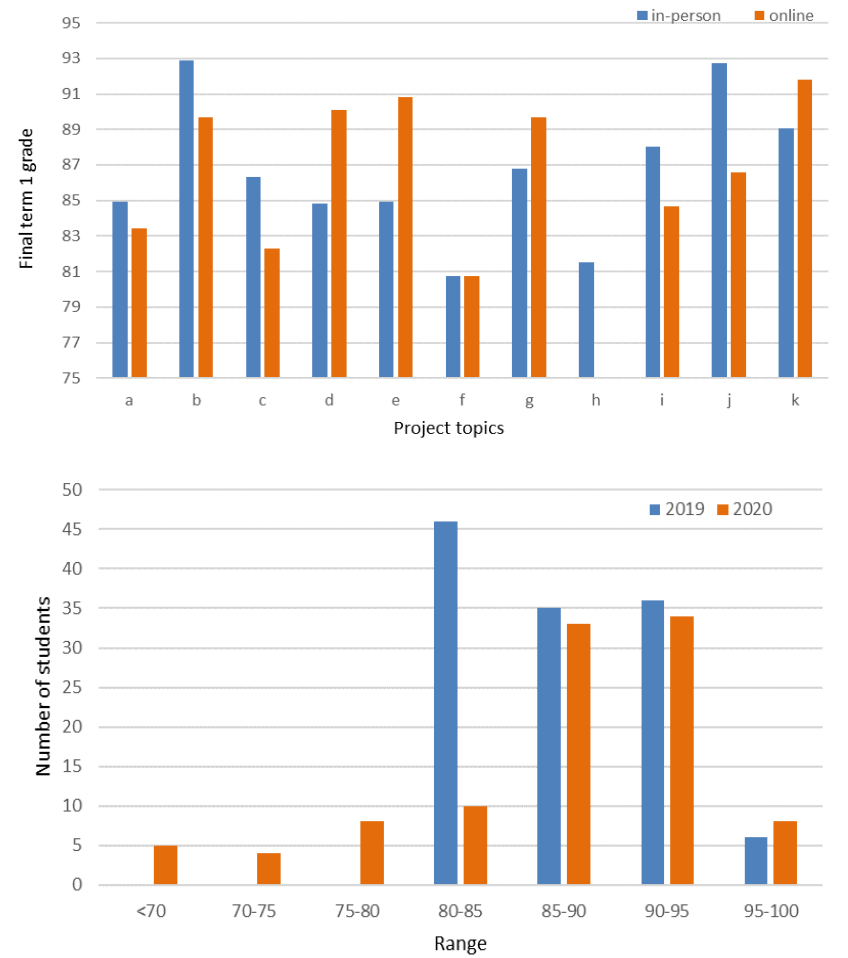

Fig. 1. Comparison of student performance for inperson $(2019$, blue) and online (2020, orange) instances of the course. Average Term 1 scores are shown broken down by lab apparatus (top, letters refer to labs listed in Table 1), and by grade tier (bottom).

and added stress of having to clearly communicate with the Operator rather than simply doing the experimental work themselves. It should be noted that the latter was expected and included in the course design as an explicit learning objective and opportunity to gain this type of experience. Several students also lamented the loss of the hands-on experience normally associated with labs. Some selected student comments illustrating these themes are shown below.

"I thought it was a very creative way to solve the online learning challenge for lab experiments. I certainly appreciated framing it in terms of us as engineers giving instructions to operators in the field, which felt applicable to our future careers. "

"I find it relatable to what I would be doing in the field. Personally, I'm not a big fan of having to be in the lab for
4 hours minimum. [In this course] I've learned how to design/structure my own experiment well in order to harness accurate and valid results. I've also learned how to create work instruction and communicate well with the TAs (operator in the field). "

"The process of having to thoroughly plan out what the operator will do before the lab was quite useful. What is unfortunate is that without previous in person lab experience, it is hard to tell the operator what to do. Example: if you have never used a $\mathrm{pH}$ probe before, how are you supposed to direct the operator to calibrate with buffer solutions and ensure that the probe has adequate electrolyte solution in it?"

"Good to establish good communication skills and technical writing skills. Hard because the lab skill portion was lost."

Interestingly, although a majority of students (26 out of 42) perceived online learning to be a positive experience in general (Q7), they preferred in person labs if given the choice (Q9), and the latter question generated the largest proportion of negative responses of any survey question. The reasons provided for preferring in-person labs are not surprising, focusing on the desire for hands-on experience working with and troubleshooting processes themselves. The main reasons for the preference for online labs for a significant percentage of the class again included lower time commitment of online labs compared to "long and uneventful [in-person] lab sessions" (presumably comparing their experience to the traditional in-person $2^{\text {nd }}$ and $3^{\text {rd }}$ year lab courses the respondents took previously), and a perception that they have gotten everything they could have out of hands-on lab experiences by the time they reached their senior year. The latter perhaps suggests some disconnect between the learning experience designed for by lab instructors, and students' perception of the benefits received taking those courses. Selected comments on online versus in-person preferences are included below.

"I would prefer to do the labs in person as I feel as though projects in the CHBE curriculum are, most of the time, very theoretical. The only way to get hands-on experience was through our labs, so not having that experience keeps us from developing practical lab skills and becoming more comfortable troubleshooting when issues arise in the lab. At the same time, performing the labs in person in groups has always been a fun experience learning to operate the equipment ourselves and see how the experiment progresses first-hand."

"I prefer the online labs. If I'm honest, I don't think our labs are equipped for us to do super cool stuff in the lab. Students aren't stretching too far, in their technical 


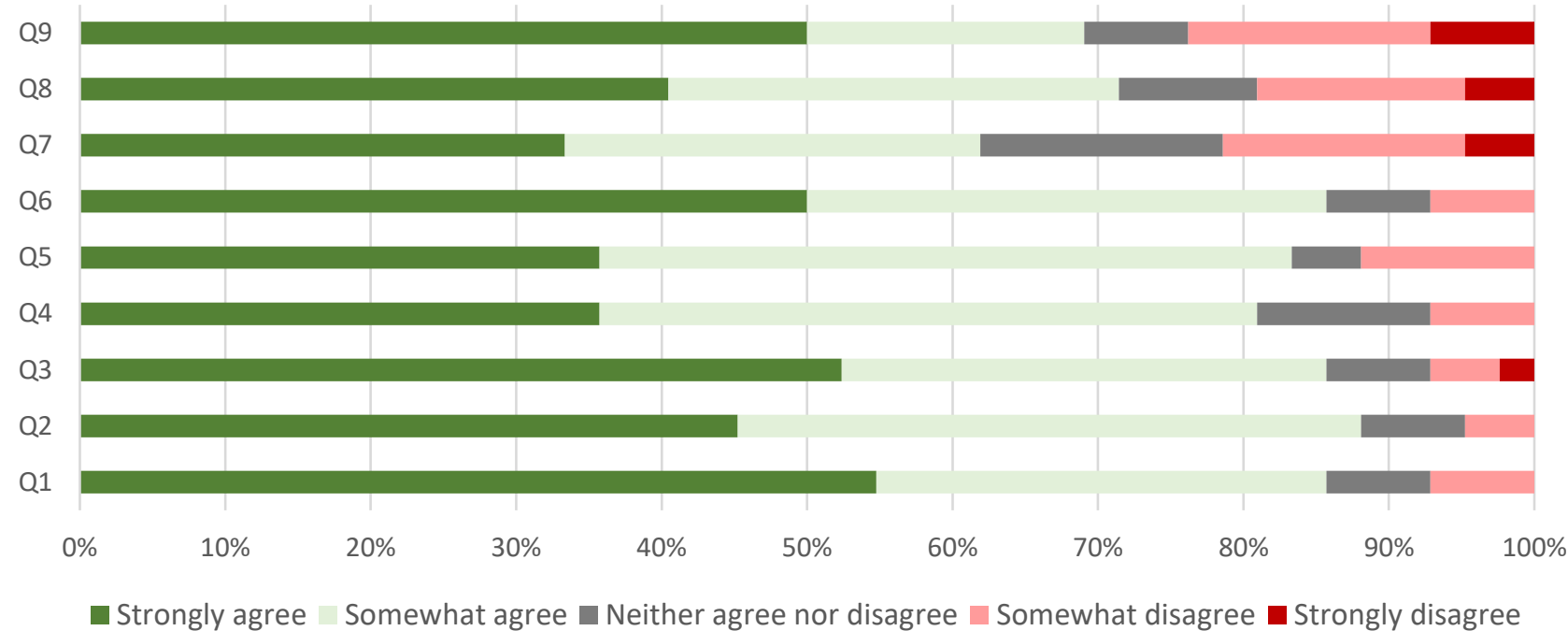

Fig. 2. Summary of responses to Likert-scale questions. Questions listed in Table 2.

skills and I don't think there are any lab practices we need to have in the field that we haven't acquired by 4th year. So, the fact that the online format provides us with practical experience to develop our soft skills (which all the employers at the career fair said trumped technical skill), provides us with experience that is valuable in the job market and will improve employment outcomes.

"I felt like we got more reliable data, because the TA's were more experienced in laboratory practice. This is also useful in our professional practice - the truth is, we aren't going to be spending a lot of time in labs taking measurements, but, more likely, will be analyzing data collected by others. I also think it's important that we learn to troubleshoot from a distance - very often we will not be working in the same physical space as the operators, so knowing what to look for in data is an important skill to develop."

Table 3 shows a comparison of the survey results collected from the in-person (2019/2020) and online (2020/2021) cohorts taking the course. We can directly compare student perception of knowledge acquisition (Q2), skill development (Q3), interest level in the particular lab assigned (Q4), increase in their confidence in engineering skills (Q5), and ease of collaboration with peers (Q8). Although we see a significant drop in all categories in terms of average Likert scores except for Q5 in Table 3, the percentage of students in the online course expressing positive opinions in each of the question represents a significant majority of the class, if a slightly smaller one than in the in-person version of the course (as more clearly outlined in Figure 3).

Although it is again difficult to directly compare answers from two different cohorts of students, there are a few points of interest to note. The biggest drop is seen, unsurprisingly, in the reported ease with which students could collaborate with each other (Q8). In an online setting active collaboration, though feasible, is much more complicated than in person, due to both the time zone differences of different team members, and the limits imposed by technology, compared to in-person collaboration (at UBC senior students have dedicated individual project rooms to work together on team deliverables for example). Also interesting is the significant drop in the students' reported interest in the subject matter of their particular lab (Q4). Although there is no obvious reason why a drop in interest would be observed given that lab assignments were performed the same way in both formats of the course, it is possible there are different learning dynamics associated with each cohort, or that the ability to extensively work hands-on with a particular process or setup (in person) increased interest in the subject matter leading to higher scores in that category since the survey. It is also unclear whether the drop in reported perception of knowledge (Q2) and skill (Q3) development is directly associated to the loss of hands-on experience, to other course design-related elements, to the aforementioned possible difference in cohort learning culture, or simply correlated to the lower reported interest in the work that the teams were assigned to. Further investigation would be warranted to draw conclusions on these questions. Finally, it is interesting to note that a similar drop was not observed in the selfreported increase in students' confidence in their engineering skills (Q5), and in fact no significant difference was measured. This may suggest that students, even if they report being less interested in the material, find a similar value or relevance of the course experience to their professional development as engineers. It is further 
interesting to note that for both the in-person and online versions of the course, there seems to be some de-coupling of one's perceived acquisition of knowledge and skills, and one's development of confidence in one's engineering skills. This seems counter-intuitive and warrants further study.

Table 3: Survey results on intended learning outcomes and engineering skills through in-person and online PBL

\begin{tabular}{|c|c|c|c|c|c|}
\hline & \multicolumn{2}{|c|}{ Mean } & \multirow[b]{2}{*}{$p$-value ${ }^{*}$} & \multicolumn{2}{|c|}{ \# of responses } \\
\hline & $\begin{array}{c}\text { In- } \\
\text { person }\end{array}$ & Online & & $\begin{array}{c}\text { In- } \\
\text { person }\end{array}$ & Online \\
\hline $\mathrm{Q} 2$ & 4.64 & 4.33 & 0.02 & 45 & 42 \\
\hline Q3 & 4.66 & 4.35 & 0.05 & 45 & 42 \\
\hline $\mathrm{Q} 4$ & 4.51 & 4.14 & 0.03 & 45 & 42 \\
\hline Q5 & 4.08 & 4.14 & 0.5 & 45 & 42 \\
\hline Q8 & 4.51 & 3.22 & 0.005 & 45 & 42 \\
\hline
\end{tabular}

p-value $<0.05$ is considered significant.

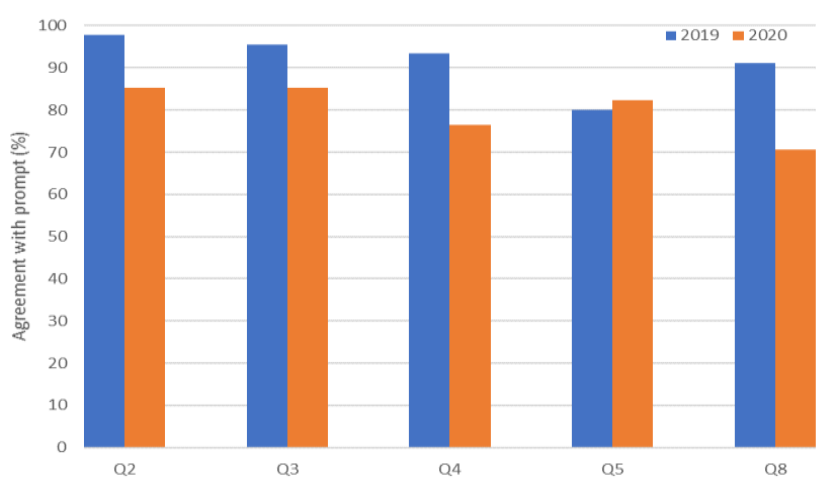

Fig. 3. Percent of students who agreed or strongly agreed with the survey prompts in both the in-person (2019, blue) and online (2020, orange) versions of the course.

Overall, the large majority of students reported this online course to be a positive experience. Although the absence of hands-on experience was again brought up by students as a drawback of this format, the new requirement of having to communicate their instructions to Operators seems to have resulted in the development of more purposeful experimental plans, and a better appreciation of the relationship between the experimental design, underlying theory, and actual processes.

"It has been a positive experience. Working alongside the TAs has worked well so far. I think this has also helped us develop skills that wouldn't have been possible otherwise (eg. effectively communicating with operator to ensure tests are performed the way we intend, even when remote) which will be very useful in our future careers."

$85 \%$ of the respondents indicated a positive experience working with TAs as the Operators (Q6) and through the open responses to Q12, indicated a comparable workload and division of labor among team members than occurs in other in-person (pre-pandemic) courses (data not shown). The main teamwork-related challenges seem again to be technological and logistical in nature and stem from limits to communication and active collaboration in the online environment.

\section{CONCLUSION}

The shift to online learning has been a challenging one for almost all courses normally delivered in person, but the transition of labs to an online setting, given their learning outcomes specifically attached to hands-on work, has perhaps been more difficult than most. This is particularly true of "wet labs" like those in chemical and biological engineering (or other similar) programs, which cannot realistically be replicated using at-home kits, and for which the simulation of elaborate unit operations, though feasible, is difficult to make worthwhile in a context requiring experimentation.

Although more challenging to shift to a virtual setting than traditional labs, the PBL format used in the senior chemical engineering laboratory course at UBC can be adapted to provide a valuable remote experience while maintaining most of the core learning outcomes and many of the benefits of problem-based pedagogical approaches. The data presented here, though presenting interesting avenues for further study, suggests that students found the experience to be a worthwhile one, and student feedback and instructor observations suggest that the model is effective and could be adapted to other contexts. It is also interesting to consider that this type of online, flexible lab in which emphasis is placed on experimental design, makes the specifics of the experimental setup unimportant, which may create opportunities for inter-institutional collaboration, whether through shared equipment or multiinstitution mixed teams, that would not be possible with traditional in-person labs, and present some interesting pedagogical options.

The responses to the survey provided by students open some interesting questions about the benefits associated with lab courses as perceived by their participants. A better understanding of any discrepancy between the benefits designed for by instructors and what students perceive as the benefits may help with the improvement of future lab courses, be they online or in person.

\section{Acknowledgements}

Heartfelt thanks are extended to Marlene Chow, Director of Academic Services in the Department of Chemical and Biological Engineering at UBC, for her steadfast willingness to support (pedagogically and financially) these new approaches to lab education. We 
would also be remiss not to thank, in no particular order, Darien Grace, Shreyas Rangan, Mahyar Montazeri, Nikoo Gharamani, Brody Frost, Fatemeh Asadi Zeidabadi, Parya Keyvani, Mahboubeh Mirzaei, Julia Antoniw, and Athanasios Kritharis, the Operators for this online lab course, who enthusiastically took on this unusual TA position, and acquitted themselves of this role with distinction. It is no exaggeration to say that they made this online lab course work.

\section{References}

[1] Z. Qiang, A.G. Obando, Y. Chen, C. Ye, Revisiting Distance Learning Resources for Undergraduate Research and Lab Activities during COVID-19 Pandemic, (2020). https://doi.org/10.1021/acs.jchemed.0c00609.

[2] L. Rice, J.L. Alquist, M. Penuliar, F. V Donato, M.M. Price, Engaging Students in a Research Methods Writing Lab Online, 48 (2021) 18-25. https://doi.org/10.1177/0098628320959954.

[3] K. Tran, A. Beshir, A. Vaze, A Tale of Two Lab Courses: An Account and Re fl ection on the Teaching Challenges Experienced by Organic and Analytical Chemistry Laboratories During the COVID-19 Period, (2020). https://doi.org/10.1021/acs.jchemed.0c00649.

[4] R. Vaez Ghaemi, G. Potvin, Experimenting with labs: Practical and pedagogical considerations for the integration of problem-based lab instruction in chemical engineering, Can. J. Chem. Eng. (2021). https://doi.org/10.1002/cjce.24136.

[5] R.L. Matz, E.D. Rothman, J.S. Krajcik, M.M.B. Holl, Concurrent Enrollment in Lecture and Laboratory Enhances Student Performance and
Retention, $\quad 49 \quad$ (2012) 659-682. https://doi.org/10.1002/tea.21016.

[6] R.M. Brockman, J.M. Taylor, L.W. Segars, V. Selke, T.A.H. Taylor, R.M. Brockman, J.M. Taylor, L.W. Segars, V. Selke, R.M. Brockman, J.M. Taylor, Student perceptions of online and inperson microbiology laboratory experiences in undergraduate medical education experiences in undergraduate medical education, Med. Educ. Online. $25 \quad$ (2020). https://doi.org/10.1080/10872981.2019.1710324.

[7] L. Phelan, A. Cullen, R. Islam, T. Kirkman, B. Mcbain, J. Pegler, T.K. Tran, T.N. Trinh, Demonstrating Adaptability: Role Modelling Multidiciplinary Learning in the Lab, Online and at Home, (n.d.) 9871834.

[8] M.J. Zhang, C. Newton, J. Grove, M. Pritzker, M. Ioannidis, Education for Chemical Engineers Design and assessment of a hybrid chemical engineering laboratory course with the incorporation of student-centred experiential learning, Educ. Chem. Eng. 30 (2020) 1-8. https://doi.org/10.1016/j.ece.2019.09.003.

[9] R.M. Felder, Designing and Teaching Courses to Satisfy, (2003). https://doi.org/10.1002/j.21689830.2003.tb00734.x.

[10] G. Potvin, Integrating Elements of Team-Based Learning and Increasing Independence in a 4thyear Lab Course to Promote the Development of Critical Thinking , Problem- Solving and Troubleshooting Skills, in: 2017 Can. Eng. Educ. Assoc. Conf., 2017: pp. 1-7.

[11] R. Vaez Ghaemi, G. Potvin, Students' Perspective: Does Problem -Based Learning Increase Ownership of One'S Education?, in: 2020 Can. Eng. Educ. Assoc., 2020: pp. 1-7. 